\title{
Panax notoginseng attenuates hypoxia-induced glycolysis in colonic mucosal epithelial cells in DSS-induced colitis
}

\author{
Wei He ${ }^{1} \wedge$, Huafeng $\operatorname{Pan}^{1} \wedge$, Ping Tao ${ }^{2}$, Jiang Lin $^{3} \wedge$, Beiping Zhang ${ }^{2}$, Shiying Wang ${ }^{1,2} \wedge$ \\ ${ }^{1}$ Science and Technology Innovation Center, Guangzhou University of Chinese Medicine, Guangzhou, China; ${ }^{2}$ Department of Gastroenterology, \\ The Second Affiliated Hospital of Guangzhou University of Chinese Medicine, Guangzhou, China; ${ }^{3}$ Department of Gastroenterology, Longhua \\ Hospital, Shanghai University of Traditional Chinese Medicine, Shanghai, China \\ Contributions: (I) Conception and design: S Wang; (II) Administrative support: J Lin, H Pan, B Zhang; (III) Provision of study materials or patients: \\ S Wang, H Pan, W He; (IV) Collection and assembly of data: S Wang, P Tao; (V) Data analysis and interpretation: W He, P Tao; (VI) Manuscript \\ writing: All authors; (VII) Final approval of manuscript: All authors. \\ Correspondence to: Shiying Wang, PhD. Science and Technology Innovation Center, Guangzhou University of Chinese Medicine, 12 Jichang Road, \\ Baiyun District, Guangzhou 510405, China. Email: yuchao387653@126.com.
}

Background: Colonic mucosal injuries are an important manifestation of ulcerative colitis (UC), which is related to hypoxia-induced glycolysis in colonic mucosal epithelial cells (cmECs). Panax notoginseng $(\mathrm{PN})$ promotes the repair of colonic mucosal injuries by inhibiting hypoxia-induced glycolysis in $\mathrm{cmECs}$; However, the mechanism by which this occurs is not completely clear. Here, we are to investigate the effects of PN on glucose metabolism in cmECs in colitis and the underlying mechanism.

Methods: A model of dextran sulfate sodium-induced colitis rats was used in this research, and the severity of colitis was assessed by pathology, disease activity index (DAI), and weight changes. The content of intracellular pyruvate, intracellular lactate, adenosine triphosphate (ATP), reactive oxygen species (ROS), mitochondrial ROS (mtROS), myeloperoxidase (MPO) activity, superoxide dismutase (SOD) activity, and inflammatory cytokines was detected by assay kits. The expression levels of proteins were detected by western blotting. The expression levels of the ATP4a gene were detected by quantitative polymerase chain reaction (QT-PCR).

Results: The colonic mucosal injuries of the colitis rats were significantly worse than those of the control group. Specifically, the hypoxia-induced glycolysis and potential of hydrogen $(\mathrm{pH})$ in the colonic lumen were increased, and the expression of ATP4a was downregulated in the colitis rats. PN $(1.0 \mathrm{~g} / \mathrm{kg})$ promoted the repair of colonic mucosal injuries, and reversed the $\mathrm{pH}$ in the colonic lumen. Further, $\mathrm{PN}$ increased the expression of ATP4a proteins, the content of ATP, and the SOD activity, and decreased the expression of pyruvate dehydrogenase lipoamide kinase isozyme and hypoxia-inducible factor 1-alpha proteins, the content of ROS, and MPO activity in cmECs in colitis. PN also increased the expression of ATP4a, cytochrome P450 family 21 subfamily a member 2, and hydroxy-delta-5-steroid dehydrogenase, 3 beta and steroid deltaisomerase 2 proteins in the mitochondria, and decreased the content of mtROS in cmECs.

Conclusions: $\mathrm{PN}$ alleviated the $\mathrm{pH}$ in the colonic lumen and hypoxia-induced glycolysis in $\mathrm{cmECs}$ by reducing the hypoxia-induced glycolysis caused by the downregulation of ATP4a protein, thereby promoting the repair of colonic mucosal injuries in colitis.

Keywords: Panax notoginseng (PN); ulcerative colitis; hypoxia; glycolysis; ATP4a protein

Submitted Jan 18, 2022. Accepted for publication Feb 18, 2022.

doi: $10.21037 /$ atm-22-566

View this article at: https://dx.doi.org/10.21037/atm-22-566

\footnotetext{
^ ORCID: Wei He, 0000-0002-4531-8448; Huafeng Pan, 0000-0003-0369-5963; Ping Tao, 0000-0002-5914-8478; Jiang Lin, 0000-00018524-6103; Beiping Zhang, 0000-0003-2979-9226; Shiying Wang, 0000-0002-1005-0381.
} 


\section{Introduction}

Ulcerative colitis (UC) is generally referred to as a chronic disorder of energy deficiency characterized by continuous mucosal injuries extending proximally from the rectum (1). The precise etiology and pathology remain unknown, but colonic mucosal injuries, which occur in the early stage of UC, are not only considered an important manifestation of UC, but also play an important role in maintaining the colonic mucosal barrier at the early stage of acute severe UC $(2,3)$. Previous studies have suggested that colonic mucosal damage may be related to colonic mucosal immune disorders, dysbiosis, hypoxia, oxidative stress, and so on $(4,5)$. Notably, it has been confirmed that hypoxia induces colonic mucosal injuries in UC (6). In a previous study, we also found that hypoxia in colonic mucosal tissues was significantly more aggravated in dextran sulfate sodium (DSS)-induced colitis rats than normal rats (7).

Colonic mucosal epithelial cells (cmECs) are the main effector cells maintaining colonic mucosal permeability (CMP). The level of glycolysis in cmECs increases significantly with aggravated hypoxia in the colitis, and is positively correlated with the severity of colonic mucosal injuries (3). Conversely, reducing hypoxia and glycolysis in cmECs has been shown to effectively promote the repair of the colonic mucosal injuries (3). However, the mechanism by which this occurs is not completely clear.

Under physiological conditions, the metabolism of glucose in cmECs is mainly based on aerobic oxidation, which can be highly regulated by multiple regulators, such as hexokinase, and pyruvate dehydrogenase lipoamide kinase isozyme 4 (PDK4) $(8,9)$. PDK4 plays a key role in the regulation of glucose metabolism, which promotes intracellular glycolysis by inhibiting the pyruvate dehydrogenase complex (PDC) (10). Further, adenosine triphosphatase (ATPase) Hydrogen ion/Potassium ion ( $\mathrm{H}+$ / $\mathrm{K}+$ ) transporting subunit alpha (ATP4a) is 1 of 2 peptide chains of $\mathrm{H}+\mathrm{K}+$ ATPase expressed in the epithelium that mediates the absorption of active $\mathrm{K}+$ in the colon (11). Our previous study showed that silencing ATP4a not only inhibited $\mathrm{H}+\mathrm{K}+$ transport, but also increased intracellular glycolysis (12). Additionally, the expression of ATP4a, which is closely related to the severity of colitis, was decreased $(13,14)$. In this study, we also found that glycolysis in cmECs was significantly increased, the expression of pyruvate dehydrogenase kinase 4 (PDK) protein was upregulated, and the expression of ATP4a was downregulated in colitis.

Panax notoginseng $(\mathrm{PN})$ is a common Chinese herbal medicine, which has been shown to attenuate colonic mucosal injury and promote mucosal repair in mouse models of colitis (7). The results of our previous research showed that $\mathrm{PN}$ attenuated hypoxia in colonic mucosa $(7,15)$, but the effect of $\mathrm{PN}$ on glucose metabolism in cmECs is unclear. Thus, in the present study, we sought to investigate the function of $\mathrm{PN}$ on hypoxia-induced glycolysis in the cmECs of colitis rats, and the mechanism by which this occurs.

We present the following article in accordance with the ARRIVE reporting checklist (available at https://atm. amegroups.com/article/view/10.21037/atm-22-566/rc).

\section{Methods}

\section{Animals}

Sprague-Dawley rats (male, 4-5 weeks, 120-140 g, SPF (Specific pathogen Free); Certificate No: 4400210019232) were obtained from Shanghai SLAC Laboratory Animal Co. Ltd. (Shanghai, China). The care and treatment of mice were in accordance with institutional guidelines. All animals used in the present study were housed in a pathogenfree environment, and experiments were performed with the approval by the Institutional Animal Care and Use Committee of Shanghai University of Traditional Chinese Medicine (No. SZY201612006), in compliance with institutional guidelines for the care and use of animals. The experiments began after 1 week of adaptive feeding. The 18 rats were randomized into the following 3 groups: (I) the normal control group (the control group, $n=6$ ); (II) the dextran sulfate sodium (DSS)-induced UC (the model group, $n=6$ ); and (III) the colitis group of rats treated with $\mathrm{PN}$ (the PN group, $1.0 \mathrm{~g} / \mathrm{kg}, \mathrm{n}=6$ ) (7) using SPSS software (Version 21.0; SPSS Inc.).

\section{Reagents}

We purchased the following: DSS (purity $>99 \%$, SigmaAldrich, St. Louis, MO, USA); ELISA kits for interleukin (IL)-4, IL-6, IL-10 and tumor necrosis factor (TNF)- $\alpha$ (R\&D Systems, Minneapolis, MN, USA); superoxide dismutase (SOD) assay kits (ab65354), myeloperoxidase (MPO) activity assay kits (ab105136), reactive oxygen species (ROS) assay kits (ab186029), ATP assay kits (ab83355), anti-rat F4/80(ab16911), cytokeratin-18 (ab133263), Platelet endothelial cell adhesion molecule-1 (CD31, ab222783), ATP4a (ab231729), Cytochrome 
P450 Family 21 Subfamily A Member 2 (CYP21A2, ab232809), Hydroxy-Delta-5-Steroid Dehydrogenase, 3 Beta and Steroid Delta-Isomerase 2 (HSD3B2, ab75710), hypoxia-inducible factor 1-alpha (HIF-1 $\alpha$; ab179483), PDK4 (ab214938), sodium potassium ATPase $(\mathrm{Na}+\mathrm{K}+$ ATPase, ab76020), voltage-dependent anion channel 1 (VDAC1/Porin, ab15895), and goat anti-rabbit antibodies, rabbit anti-mouse antibodies (Abcam, Cambridge, UK); mitochondrial ROS (mtROS) detection assay kits (Cayman Chemical Co., 701600, MI, USA); and lipofectamine 2000 transfection reagent (\#11668019, ThermoFisher, Waltham, MA, USA). The rat ATP4a gene silencing plasmid (designed by targeting NM_012509.1 gene) was constructed and synthesized by GK Gene (Shanghai, China).

\section{Assessment of SOD, ROS, MPO, and ATP levels}

The levels of SOD, ROS, MPO, and ATP in the colonic mucosa and cmECs were examined as indicators of tissue peroxidation using the appropriate assay kits as per the instructions of the manufacturer.

\section{Extraction of proteins from the mitochondria and cell membranes}

The cell membrane proteins were extracted according to the CelLytic ${ }^{\mathrm{TM}}$ MEM Protein Extraction kit (CE0050, sigma, USA), and the mitochondria were isolated using the mitochondrial isolation kit (MITOISO2, sigma, USA) and lysed for proteins.

\section{DSS-induced colitis, the preparation and intervention of $P N$ in vivo, the weight, DAI, MVD and colonic mucosal tissue analysis, $P H$ determination, quantitative $R T$ - PCR, isolation of colonic mucosal cells, cultures and interventions, cell viability, $W B$}

DSS-induced colitis, the preparation and intervention of $\mathrm{PN}$ in vivo, the weight, DAI, microvessel density (MVD), and the colonic mucosal tissue analysis, cell viability, and western blotting (WB) were carried out as previously described $(7,16)$. The potential of hydrogen $(\mathrm{pH})$ analysis and quantitative polymerase chain reaction (QT-PCR) analysis were carried out as previously described (17). Lamina propria mononuclear cells (LPMCs), cmECs, and colonic mucosal microvascular endothelial cells (cmVECs) were isolated, and the cultures and interventions were carried out as previously described (18).

\section{Plasmid construction and cell transfection}

To clarify the mechanism by which ATP4a is involved in regulating glucose metabolism, the silencing plasmids of the ATP4a gene were used. Empty plasmids (E-ps) were used as the negative controls. The cmECs were seeded into 24-well plates (Corning, China). In accordance with the manufacturer's instructions, transfection was performed with Lipofectamine 2000 Transfection Reagent when the cells reached $50 \%$ to $80 \%$ confluence. The culture medium was changed after 4 to 6 hours, and the cells continued to be incubated at $37^{\circ} \mathrm{C}$ in a carbon-dioxide (CO2)-incubator for 24 hours. Next, gene expression was detected by WB. The cells were divided into the following groups: (I) the control group (un-transfected); (II) the E-p group (transfected with empty plasmid); (III) the ATP4a ${ }^{\text {si }}$ group (transfected with ATP4a silencing plasmids); and (IV) the PN + ATP4a group (ATP $4 \mathrm{a}^{\text {si }} \mathrm{cmECs}$ treated with PN).

\section{Statistical analysis}

The data are presented as the mean \pm standard error of the mean. A 1-way analysis of variance or general linear model with repeated measures was used to analyze the data sets with 3 or more groups, and the least significant difference post-hoc test was used for multiple comparisons. The student's $t$-test was used to analyze data sets with 2 groups. A P value $<0.05$ was considered significant. Only the corresponding author who performed the data statistics was aware of the group allocation at the different stages of the experiment.

\section{Results}

\section{$P N$ promoted the repair of colonic mucosal injuries in DSS-induced colitis}

Compared to the control group, the colonic mucosal inflammatory injuries were greatly increased in the model group (see Figure 1A). Additionally, the DAI scores were significantly increased and the weight of the colitis rats was decreased (see Figure 1B,1C), while the serum IL-4 and IL-10 levels were decreased, and IL- 6 and TNF- $\alpha$ serum levels were increased in the colitis rats (see Figure 1D). However, compared to the rats in the model group, PN $(1.0 \mathrm{~g} / \mathrm{kg})$ significantly increased the weight, decreased the 
A

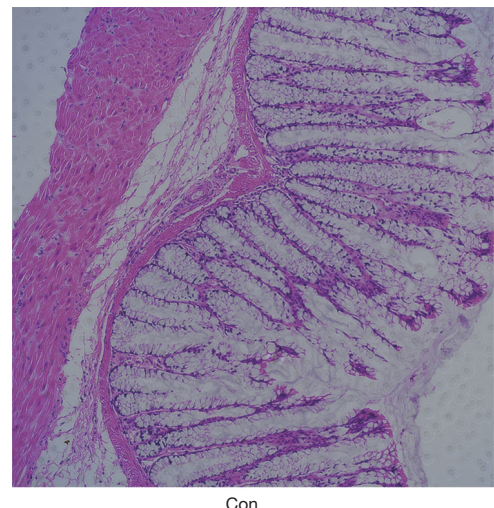

Con

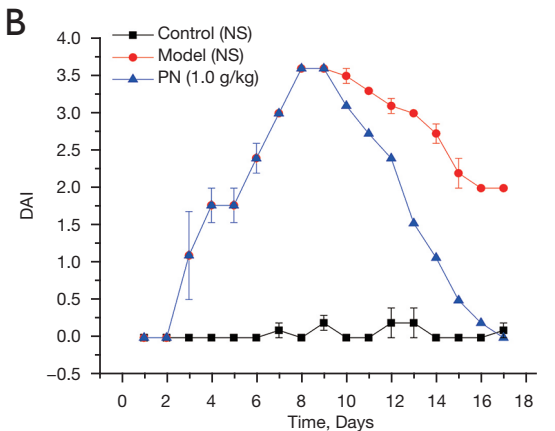

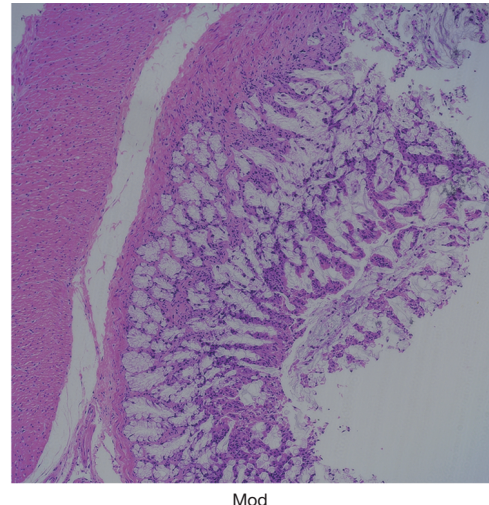

Mod

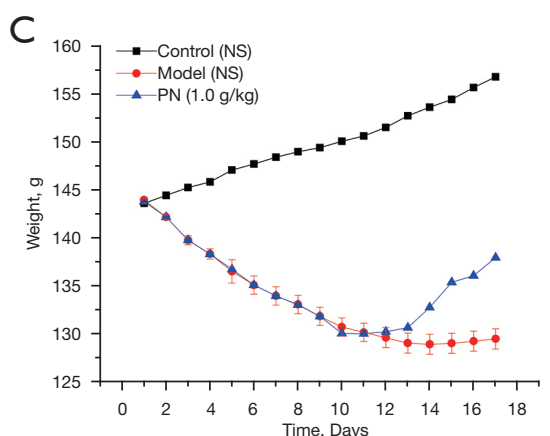

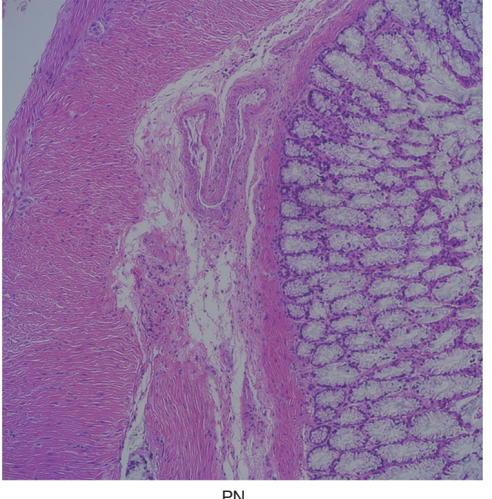

$\mathrm{PN}$

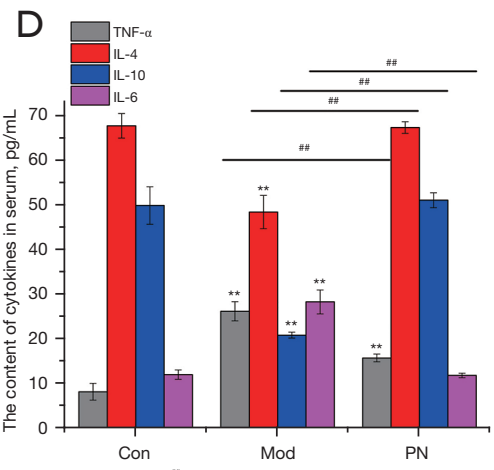

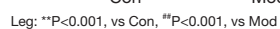

Figure 1 PN promoted the repair of colonic mucosal injuries in DSS-induced colitis. (A) Colon mucosa pathological changes (H\&E, 400x); (B) DAI core; (C) the weight of the colitis rats; (D) the content of serum IL-4, IL-10, IL-6, and TNF- $\alpha$. Con, Control group; Mod, Model group; PN, Model group treated with PN. PN, panax notoginseng; NS, normal saline; DSS, dextran sulfate sodium; DAI, disease activity index; IL, interleukin; TNF- $\alpha$, tumor necrosis factor $\alpha$.

DAI scores, and limited the colonic mucosal inflammation of the colitis rats by decreasing the serum levels of IL-6 and TNF- $\alpha$, increasing the serum levels of IL- 4 and IL-10, and promoting the repair of colonic mucosal injuries (see Figure $1 A-1 D$ ). Thus, PN significantly promoted the repair of colonic mucosal ulcers.

\section{PN attenuated colonic mucosal bypoxia and glycolysis in colitis}

In the model group, the SOD activity was decreased, but the expression of ATP4a, HIF-1 $\alpha$, ROS, and MPO activity was increased in the colonic mucosa (see Figure 2A-2E). Additionally, the concentration of intracellular pyruvate and lactate was significantly increased in the model group compared to that of the control group (see Figure 2F,2G). However, both the increase of the expression of ATP4a, HIF- $1 \alpha$, ROS, and MPO activity, and the decrease of SOD activity was limited to a certain extent in the group treated with PN (i.e., the PN group) (see Figure $2 A-2 E$ ). Further, the concentration of intracellular pyruvate and lactate was significantly decreased in the PN group (see Figure 2F,2G). Notably, the $\mathrm{PH}$ in the colonic lumen was significantly more elevated in the model group than the control group (see Figure $2 H$ ). Thus, PN significantly ameliorated colonic mucosal hypoxia and glycolysis in the colonic mucosa of the colitis rats.

\section{PN suppressed hypoxia-induced glycolysis in cmECs}

To explore the effects of glycolysis on colonic mucosal injuries, the cmECs were isolated. The results showed that the cell viability of the cmECs isolated from the model group was significantly decreased (see Figure $3 A$ ). Additionally, the SOD activity was decreased, but the expression of caspase- 3 and HIF-1 $1 \alpha$ protein, the content of ROS, and MPO activity were increased in the cmECs isolated from the model group (see Figure $3 B-3 F$ ). Further, 

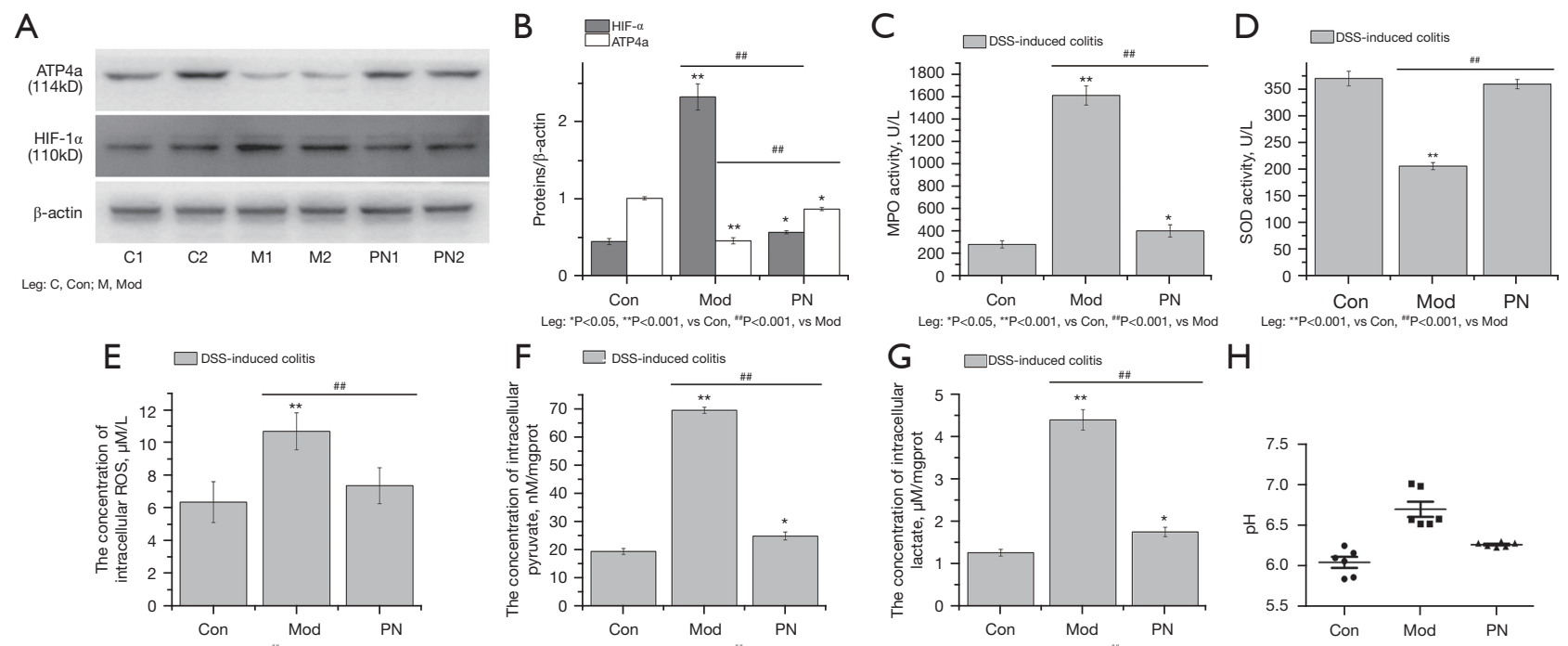

$$
\text { Leg: " } \mathrm{P}<0.001 \text {, vs Con, " } \mathrm{P}<0.001 \text {, vs Mod }
$$

$$
\text { Leg: }{ }^{*}<<0.05,{ }^{*} P<0.001 \text {, vs Con, " } P<0.001 \text {, vs Mod }
$$

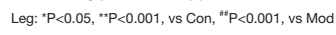

$\mathrm{H}$

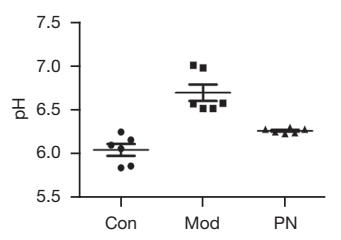

Figure 2 PN attenuated colonic mucosal hypoxia and aerobic glycolysis in colitis. (A-E) The SOD activity, the expression of ATP4a and HIF-1 $\alpha$ proteins, The content of ROS and MPO activity in the colonic mucosa; (F,G) the concentration of intracellular pyruvate and lactate; $(\mathrm{H})$ the $\mathrm{pH}$ in the colonic lumen. Con, Control group; Mod, Model group; PN, Model group treated with PN. SOD, Superoxide dismutase; ATP4a, ATPase H+/K+ Transporting Subunit Alpha; HIF-1 $\alpha$, hypoxia-inducible factor 1-alpha; ROS, reactive oxygen species; $\mathrm{MPO}$, myeloperoxidase; $\mathrm{pH}$, potential of hydrogen.

the concentration of intracellular pyruvate and lactate was significantly more increased in the cmECs isolated from the model group than those isolated from the control group (see Figure $3 G, 3 H$ ). However, the cell viability of the cmECs isolated from the $\mathrm{PN}$ group was significantly more increased than that of the cmECs isolated from the model group (see Figure 3A). Additionally, the expression levels of caspase-3 and HIF- $1 \alpha$ protein, the content of ROS, and MPO activity were significantly decreased, and the SOD activity was significantly increased in the cmECs isolated from the PN group compared to those isolated from the model group (see Figure $3 B-3 F)$. The concentration of intracellular pyruvate and lactate was also significantly decreased in the PN group (see Figure 3G,3H).

\section{PN upregulated the expression of ATP4a in cmECs}

As discussed above, the expression of ATP4a protein was significantly decreased in the colonic mucosa of the model group. In this study, cmECs, LPMC, and cmVECs were isolated from the control and model group. The expression of ATP4a was significantly more downregulated in the cmECs than the LPMCs and cmVECs (see Figure 4A-4C). Additionally, the expression of ATP4a protein in both the cell membranes and mitochondria were significantly downregulated in the cmECs isolated from the model group (see Figure 4D,4E).

The expression of ATP4a was more decreased and the expression of PDK4 protein was more increased in the cmECs isolated from the model group than those of the control group, while the expression of ATP4a was more upregulated, and the expression of PDK4 protein was more decreased in the cmECs isolated from the PN group than those isolated from the model group (see Figure $4 F-4 H$ ).

\section{PN ameliorated glycolysis by upregulating the expression of ATP4a protein in cmECs}

As discussed above, the glycolysis in the cmECs was increased in the model group, and the expression of ATP4a protein was decreased, but these findings were reversed in the PN group. In this study, the cmECs were isolated from normal rats (male, aged 3-4 weeks old) and cultured in vitro.

ATP $4 a^{\text {si }}$ plasmid was used to regulate the expression of ATP4a in the cmECs. The results showed that the expression of ATP4a protein in cmECs was significantly downregulated by ATP4a $\mathrm{a}^{\text {si }}$ plasmid (S-p3) (see Figure 5A). The cell viability was not significantly decreased, but the expression of PDK4 protein, and the concentration of intracellular pyruvate and lactate were increased in $\mathrm{ATP} 4 \mathrm{a}^{\mathrm{si}}$ cmECs (see Figure 5B-5F). Conversely, PN (0.3 g/L) significantly increased the cell viability of cmECs and 


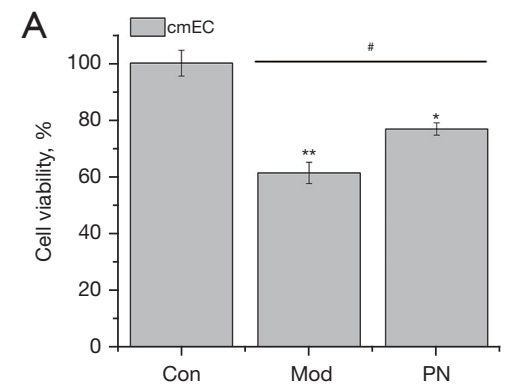

Leg: ${ }^{\star} \mathrm{P}<0.05,{ }^{\star \star} \mathrm{P}<0.001$, vs Con; ${ }^{*} \mathrm{P}<0.05$, vs Mod

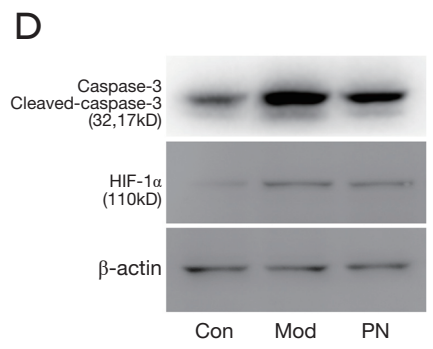

Leg: cmEC isolated from the Control, Model, $\mathrm{PN}$ group

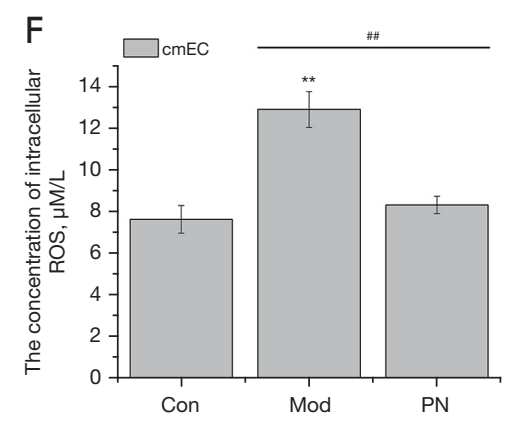

Leg: ${ }^{\star \star} \mathrm{P}<0.001$, vs Con; ${ }^{* \#} \mathrm{P}<0.001$, vs Mod

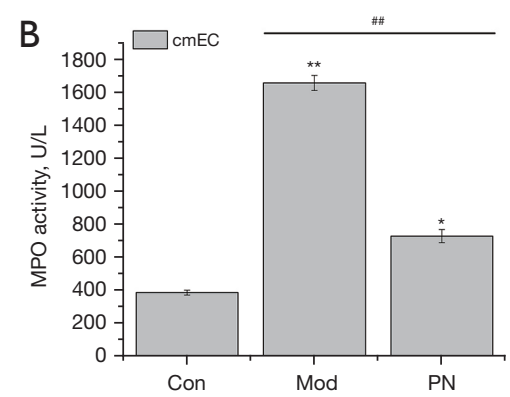

Leg: ${ }^{*}<<0.05,{ }^{* *} \mathrm{P}<0.001$, vs Con; ${ }^{* *} \mathrm{P}<0.001$, vs Mod
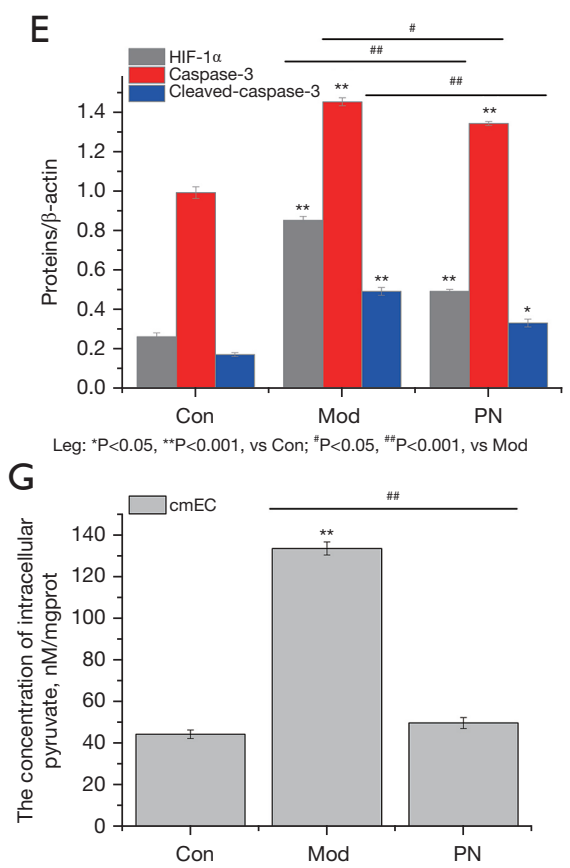

Leg: ${ }^{* *} \mathrm{P}<0.001$, vs Con; ${ }^{* *} \mathrm{P}<0.001$, vs Mod

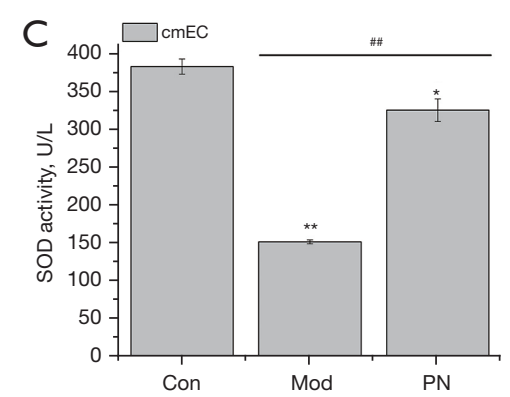

Leg: ${ }^{*} \mathrm{P}<0.05,{ }^{* *} \mathrm{P}<0.001$, vs Con; ${ }^{* *} \mathrm{P}<0.001$, vs Mod

Figure 3 PN suppressed aerobic glycolysis and hypoxia in cmECs. (A) The cell viability of cmECs; (B-F) the SOD activity, the expression of caspase-3 and HIF-1 $\alpha$ protein, the content of ROS and MPO activity; $(\mathrm{G}, \mathrm{H})$ the concentration of intracellular pyruvate and lactate. PN, panax notoginseng; cmEC, colonic mucosal epithelial cells; SOD, superoxide dismutase; HIF-1 $\alpha$, hypoxia-inducible factor 1-alpha; ROS, reactive oxygen species; MPO, myeloperoxidase.

ATP $4 \mathrm{a}^{\text {si }} \mathrm{cmECs}$, decreased the expression of PDK4 protein, and the concentration of intracellular pyruvate and lactate, and upregulated the expression of ATP4a (see Figure 5B-5F).

\section{PN activated mitochondrial aerobic oxidation by upregulating the expression of ATP4a in the mitochondria in cmECs}

The expression of ATP4a protein was downregulated in the mitochondria of the cmECs isolated from the model group. Additionally, the content of ATP was significantly decreased and the expression of ATP4a protein was downregulated in the colonic mucosa and cmECs isolated from the model group (see Figure $6 A, 6 B$ ). The content of ROS and mtROS in the ATP4 $\mathrm{a}^{\mathrm{si}} \mathrm{cmECs}$ increased significantly with the expression of ATP4a, CYP21A2, and HSD3B2 proteins in the mitochondria downregulated (see Figure 6C-6F). Conversely, the content of ATP in the colonic mucosa and cmECs isolated from the $\mathrm{PN}$ group was increased compared to that of the model group (see Figure 6A,6B). Additionally, PN significantly decreased the content of ROS and mtROS in ATP4 $\mathrm{a}^{\text {si }} \mathrm{cmECs}$, and increased the expression of ATP4a, CYP21A2, and HSD3B2 protein in the mitochondria (see Figure 6C-6F). 
A

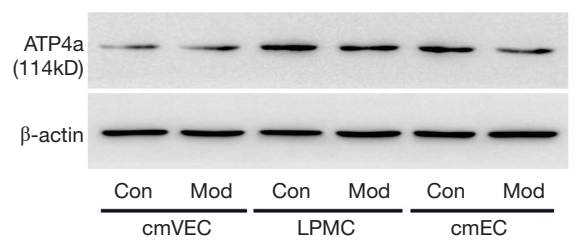

D

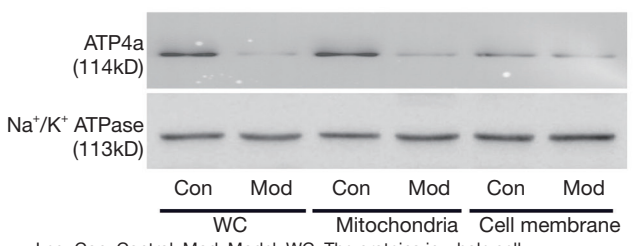

Leg: Con, Control; Mod, Model; WC, The proteins in whole cell

\section{$\mathrm{F}$}

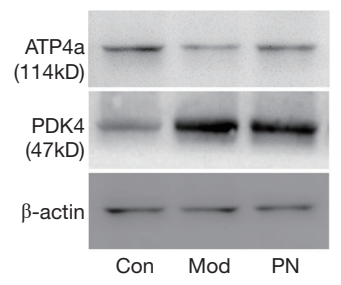

Leg: cmEC isolated from the Control, Model, PN group
B

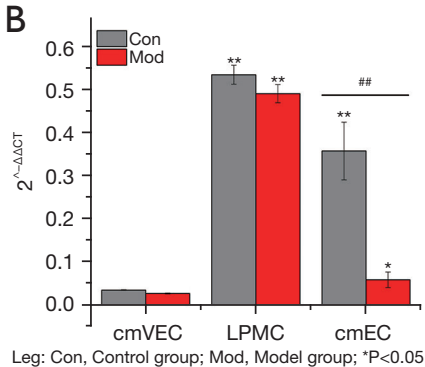

Leg: Con, Control group; Mod, Model group; ${ }^{*} \mathrm{P}<0.05$
${ }^{*} \mathrm{P}<0.001$, vs cmVEC; ${ }^{*{ }^{*} \mathrm{P}}<0.001$, Con vs Mod

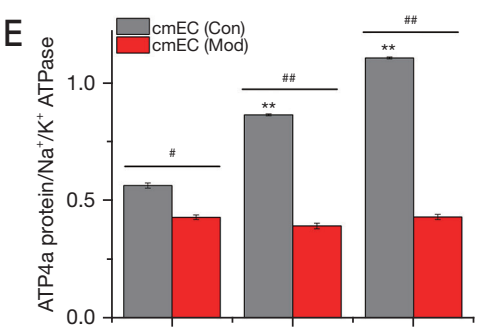

WC Mito $\quad \mathrm{CM}$

Leg: Con, Control group; Mod, Model group; ${ }^{* *} \mathrm{P}<0.001$, vs cmVEC; ${ }^{*} \mathrm{P}<0.05,{ }^{*{ }^{*} \mathrm{p}}<0.001$, Con vs Mod
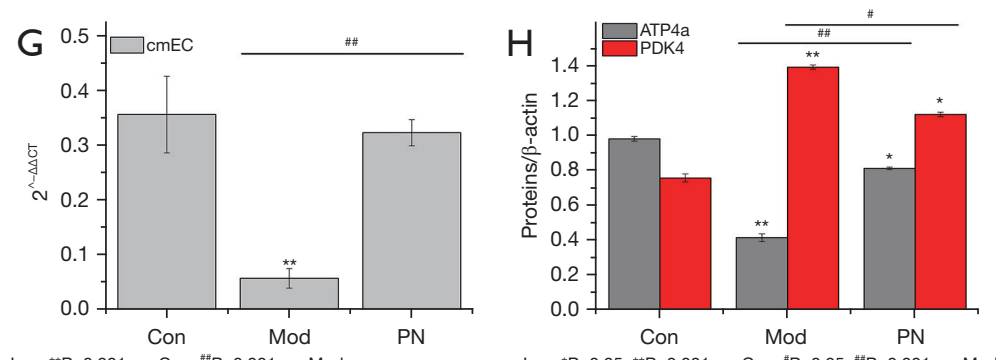

Leg: ${ }^{*} \mathrm{P}<0.05,{ }^{* *} \mathrm{P}<0.001$, vs Con; ${ }^{*} \mathrm{P}<0.05,{ }^{* *} \mathrm{P}<0.001$, vs Mod

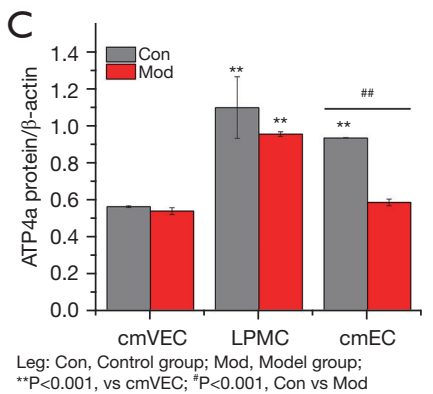

Figure 4 PN upregulated the expression of ATP4a in cmECs. (A-C) The expression of ATP4a in cmECs, LPMC, and cmVECs; (D,E) the expression of ATP4a protein in the cell membranes and mitochondria; $(\mathrm{F}-\mathrm{H})$ the expression of ATP4a and PDK4 protein in cmECs. PN, panax notoginseng; ATP4a, ATPase H+/K+ Transporting Subunit Alpha; cmEC, colonic mucosal epithelial cells; LPMC, lamina propria mononuclear cells; cmVECs, colonic mucosal microvascular endothelial cells; PDK4, pyruvate dehydrogenase lipoamide kinase isozyme 4.

\section{Discussion}

$\mathrm{PN}$ is a common herbal medicine with various activities, and has been widely used to treat diabetes, osteoporosis, and colitis in China (19-21). In our previous studies, PN has been used to treat $\mathrm{UC}$, and its promotion of the repair of mucosal injury and anti-inflammatory responses have been widely proven (7,22); PN also improves hypoxia in the colonic mucosa by reducing colonic mucosal microvascular injuries in colitis (7). Similarly, the results of this study showed that PN alleviated hypoxia in the colonic mucosa and promoted the repair of colonic mucosal injuries in colitis.

CmECs are the main cells that play a critical role in maintaining CMP. The maintenance of the structure and function of cmECs depends on normal intracellular energy metabolism, especially the aerobic oxidation of glucose. Hypoxia in the colonic mucosa significantly inhibits the normal aerobic oxidation of glucose and increases the anaerobic glycolysis in cmECs (23). Additionally, research has shown that with the aggravation of hypoxia in the colonic mucosa, the apoptosis and necrosis of cmECs increase significantly, both of which are positively correlated to the severity of UC $(24,25)$. This study showed that intracellular glycolysis in cmECs was significantly enhanced, and hypoxia was aggravated in the colonic mucosa in colitis. Further, PN improved the cell viability of $\mathrm{cmECs}$, inhibited intracellular glycolysis, and ameliorated hypoxia in the colonic mucosa, especially in cmECs in colitis. Thus, PN reduced the colonic mucosal injuries by inhibiting hypoxiainduced intracellular glycolysis. 
A

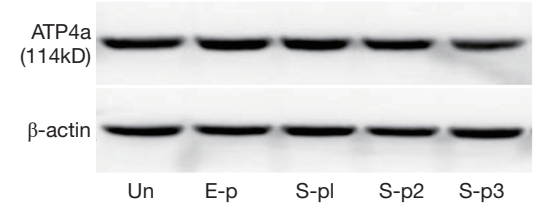

Leg: Un, untransfected; E-p, empty plasmid; S-p, silencing plasmid

C
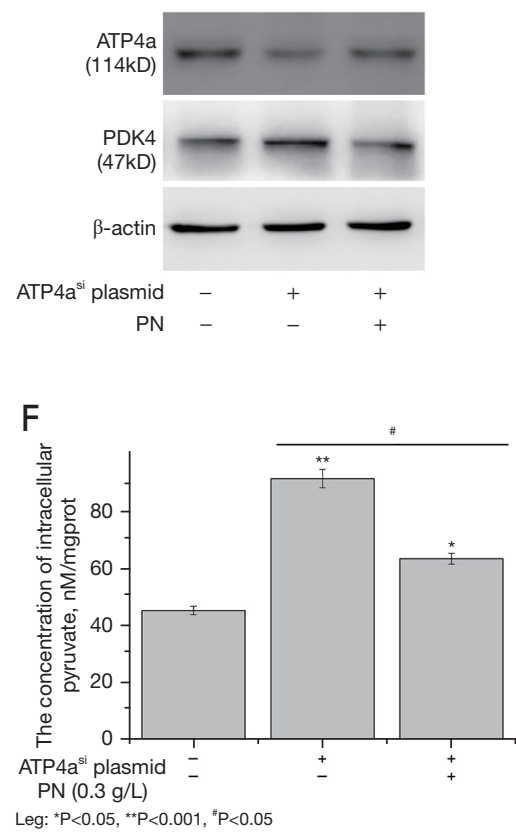

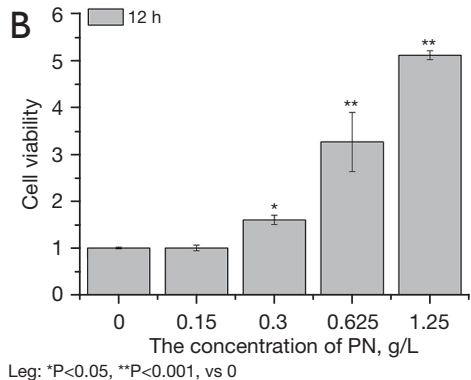

D
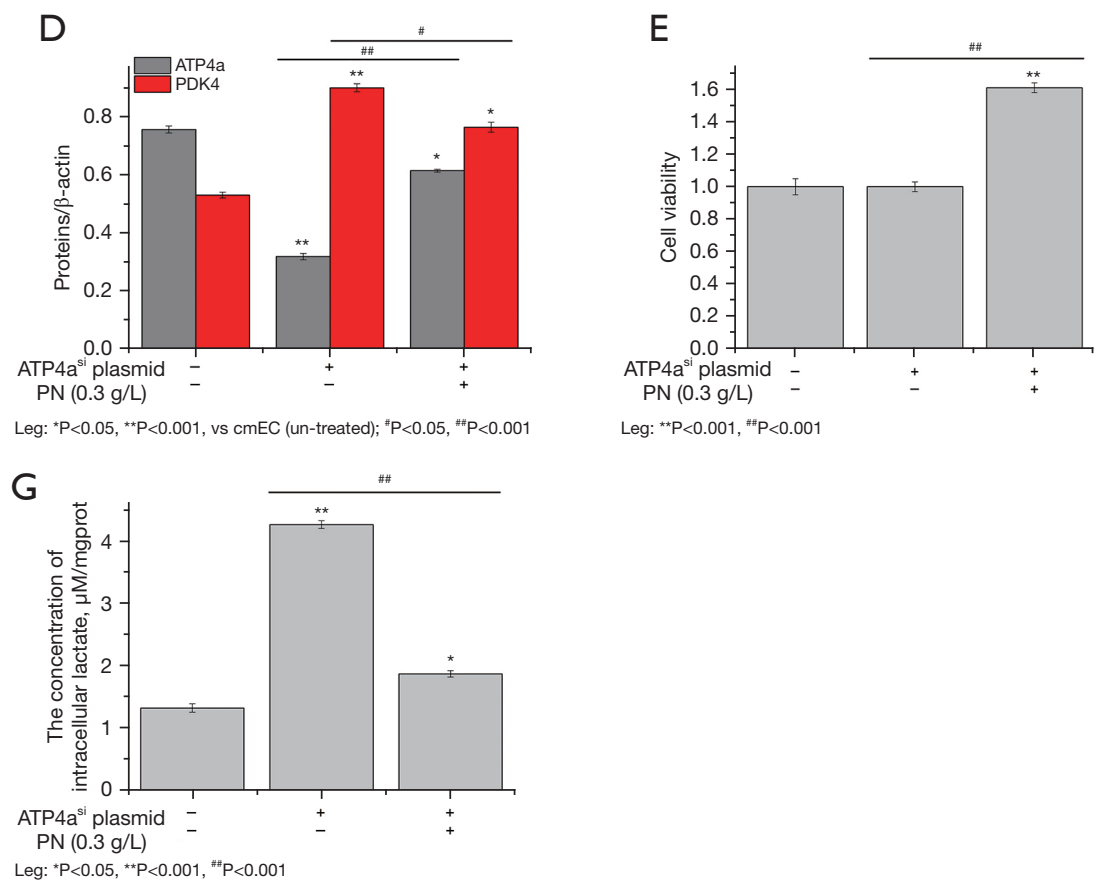

Figure $5 \mathrm{PN}$ ameliorated glycolysis by upregulating the expression of ATP4a protein in cmECs. (A) The expression of ATP4a protein in cmECs was significantly downregulated by ATP4a ${ }^{\text {si }}$ plasmid (S-p3); (B) the cell viability; (C-F) the expression of PDK4 protein, the concentration of intracellular pyruvate and lactate. PN, panax notoginseng; ATP4a, ATPase H+/K+ Transporting Subunit Alpha; cmEC, colonic mucosal epithelial cells; PDK4, pyruvate dehydrogenase lipoamide kinase isozyme 4.

The aerobic oxidation of glucose is an ordered process catalyzed by a series of enzymes. Thus, the occurrence of intracellular glycolysis might be related to the abnormal synthesis and function of related enzymes involved in the aerobic oxidation of glucose, such as PDK4, PDC, CYP21A2, and HSD3B2 $(10,26,27)$. Notably, research has shown that PDK4 significantly promotes intracellular glycolysis by inhibiting PDC, and thereby regulates metabolite flux through the tricarboxylic acid cycle, downregulates aerobic respiration, and inhibits the formation of acetyl-coenzyme A from pyruvate (10). Our results showed that hypoxia and intracellular glycolysis were significantly increased and the expression of PDK4 protein was upregulated in the cmECs of the colitis rats. Conversely, PN reduced hypoxia-induced intracellular glycolysis by downregulating the expression of PDK4 protein in cmECs.

Additionally, ATP4a, a peptide chain of $\mathrm{H}+\mathrm{K}+$ ATPase expressed in the epithelium that mediates the absorption of active $\mathrm{K}+$ in the colon (11), affects the regulation of the intracellular glucose metabolism. The results of previous studies suggest that the expression of ATP4a is significantly inhibited in colitis $(13,14)$. In a previous study, we also found that silencing ATP4a not only inhibits $\mathrm{H}+\mathrm{K}+$ transport but also increases intracellular glycolysis (12). In this study, our results showed that the expression of 
A

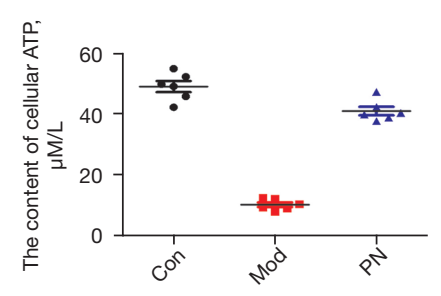

D

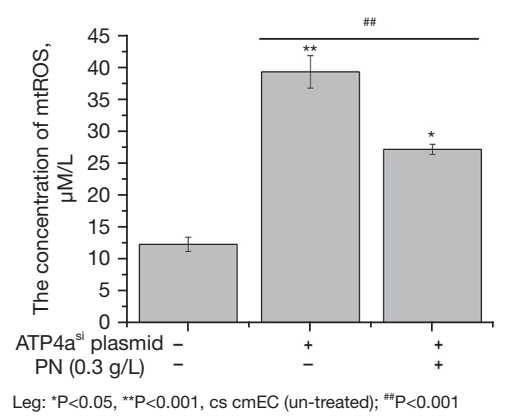

B

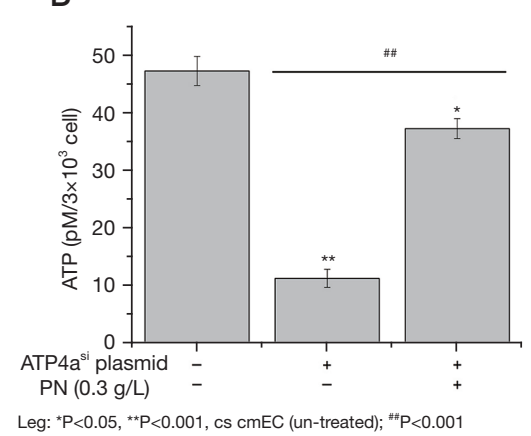

$\mathrm{E}$

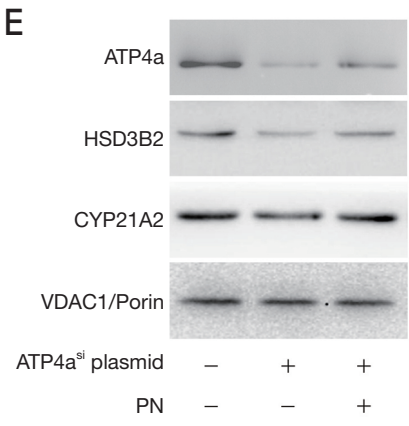

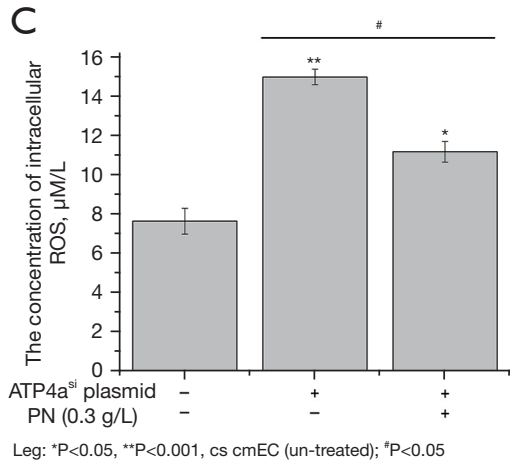

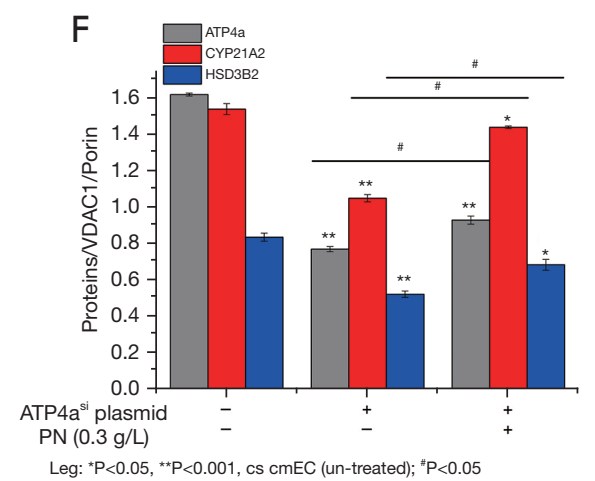

Figure $6 \mathrm{PN}$ activated the mitochondrial aerobic oxidation by upregulating the expression of ATP4a in the mitochondria in cmECs. (A) The content of ATP; (B) the expression of ATP4a protein; (C,D) the content of ROS and mtROS; (E,F) the expression of ATP4a, CYP21A2, and HSD3B2 proteins in the mitochondria. PN, panax notoginseng; ATP4a, ATPase H+/K+ Transporting Subunit Alpha; cmEC, colonic mucosal epithelial cells; ATP, adenosine triphosphate; ROS, reactive oxygen species; mtROS, mitochondrial ROS; CYP21A2, Cytochrome P450 Family 21 Subfamily A Member 2; HSD3B2, hydroxy-delta-5-steroid dehydrogenase, 3 beta and steroid delta-isomerase 2.

ATP4a was significantly downregulated with intracellular glycolysis and $\mathrm{pH}$ in colon increased in colitis, and the increased of $\mathrm{pH}$ in colon might influence the distribution of gut microbiota; however, $\mathrm{PN}$ decreased intracellular glycolysis and $\mathrm{pH}$ in the colon by increasing the expression of ATP4a in cmECs. Additionally, the decrease in the expression of ATP4a in the mitochondria was much more significant than that in the cell membrane in cmECs. Further, silencing ATP4a in cmECs increased intracellular glycolysis, and downregulated the expression of ATP4a in the mitochondria, which significantly increased the content of mtROS and decreased the content of ATP in cmECs.

CYP21A2 and HSD3B2 are important enzymes that regulate the aerobic oxidation of glucose in the mitochondria. The results of previous studies suggest that the expression of CYP21A2 protein is significantly downregulated in the colonic mucosa in colitis (26), and downregulating the expression of CYP21A2 protein in the mitochondria in cmECs significantly inhibits the aerobic oxidation of glucose, and also increases the content of ATP (26). HSD3B2 is a 2-substrate enzyme, which binds to the cofactor nicotinamide adenine dinucleotide (NAD), which is involved in the transport of $\mathrm{H}$ in the mitochondria (28). Interestingly, our results showed that the expression levels of CYP21A2 and HSD3B2 proteins in the mitochondria in cmECs were significantly downregulated in colitis, and the expression level of ATP4a was downregulated and the content of ATP decreased.

The decrease of the expression of ATP $4 a$ in the mitochondria was much more significant than that in the cell membrane in cmECs. Silencing ATP4a in cmECs upregulated the expression of PDK4, and increased intracellular glycolysis in vitro. Further, downregulating the expression of ATP4a in the mitochondria in cmECs significantly downregulated the expression of CYP21A2 and HSD3B2 proteins, decreased the content of intracellular ATP, and increased the content of mtROS. Conversely, PN decreased the content of mtROS, increased the content 
of ATP, and upregulated the expression of CYP21A2 and HSD3B2 proteins in the mitochondria in cmECs by downregulating the expression of ATP4a in colitis.

In summary, PN alleviated hypoxia-induced glycolysis in cmECs by reducing the abnormal aerobic oxidation of glucose caused by the downregulation of ATP4a protein, thereby promoting the repair of colonic mucosal injuries in colitis. Additionally, $\mathrm{PN}$ also corrected the elevated $\mathrm{pH}$ in the colonic lumen caused by the downregulation of ATP4a protein in cmECs. However, there are still some unresolved problems in the present study that require further study. First, the mechanism underlying the decreased expression of ATP4a in the colonic mucosa of rats with colitis is unclear. Second, research needs to be conducted to determine the active ingredients of $\mathrm{PN}$, and the mechanisms by which $\mathrm{PN}$ regulates the expression of ATP4a in the mitochondria of cmECs. Third, the effect of increased $\mathrm{pH}$ in the colonic lumen caused by the decrease in ATP4a protein on the distribution of the gut microbiota is unclear. Finally, because of the lack of ATP-deficient knockout mice in this study, the results could not be confirmed in vivo. These issues should be explored in future studies.

\section{Acknowledgments}

The authors would like to thank all the technical staff who provided help with this study.

Funding: The study was supported by the National Natural Science Foundation of China (No. 81904106), Major Scientific Research Projects at Provincial Level at Guangdong General University (No. 2018KQNCX044) and scientific special expenditure of Guangdong Provincial Hospital of Traditional Chinese Medicine (No. YN2020QN15).

\section{Footnote}

Reporting Checklist: The authors have completed the ARRIVE reporting checklist. Available at https://atm. amegroups.com/article/view/10.21037/atm-22-566/rc

Data Sharing Statement: Available at https://atm.amegroups. com/article/view/10.21037/atm-22-566/dss

Conflicts of Interest: All authors have completed the ICMJE uniform disclosure form (available at https://atm. amegroups.com/article/view/10.21037/atm-22-566/coif). The authors have no conflicts of interest to declare.
Ethical Statement: The authors are accountable for all aspects of the work in ensuring that questions related to the accuracy or integrity of any part of the work are appropriately investigated and resolved. All animal experiments were performed with the approval by the Institutional Animal Care and Use Committee of Shanghai University of Traditional Chinese Medicine (No. SZY201612006), in compliance with institutional guidelines for the care and use of animals.

Open Access Statement: This is an Open Access article distributed in accordance with the Creative Commons Attribution-NonCommercial-NoDerivs 4.0 International License (CC BY-NC-ND 4.0), which permits the noncommercial replication and distribution of the article with the strict proviso that no changes or edits are made and the original work is properly cited (including links to both the formal publication through the relevant DOI and the license). See: https://creativecommons.org/licenses/by-nc-nd/4.0/.

\section{References}

1. Kobayashi T, Siegmund B, Le Berre C, et al. Ulcerative colitis. Nat Rev Dis Primers 2020;6:74.

2. Seidelin JB, Bahl MI, Licht TR, et al. Acute Experimental Barrier Injury Triggers Ulcerative Colitis-Specific Innate Hyperresponsiveness and Ulcerative Colitis-Type Microbiome Changes in Humans. Cell Mol Gastroenterol Hepatol 2021;12:1281-96.

3. Eissa N, Hussein H, Tshikudi DM, et al. Interdependence between Chromogranin-A, Alternatively Activated Macrophages, Tight Junction Proteins and the Epithelial Functions. A Human and In-Vivo/In-Vitro Descriptive Study. Int J Mol Sci 2020;21:7976.

4. Yang Q, Wang Y, Jia A, et al. The crosstalk between gut bacteria and host immunity in intestinal inflammation. J Cell Physiol 2021;236:2239-54.

5. Bilski J, Mazur-Bialy A, Wojcik D, et al. Effect of Forced Physical Activity on the Severity of Experimental Colitis in Normal Weight and Obese Mice. Involvement of Oxidative Stress and Proinflammatory Biomarkers. Nutrients 2019;11:1127.

6. Sun L, Li T, Tang H, et al. Intestinal Epithelial CellsDerived Hypoxia-Inducible Factor-1 $\alpha$ Is Essential for the Homeostasis of Intestinal Intraepithelial Lymphocytes. Front Immunol 2019;10:806.

7. Wang SY, Tao P, Hu HY, et al. Effects of initiating time and dosage of Panax notoginseng on mucosal 
microvascular injury in experimental colitis. World J Gastroenterol 2017;23:8308-20.

8. Cui Z, Wang Q, Deng MH, et al. LncRNA HCG11 promotes 5-FU resistance of colon cancer cells through reprogramming glucose metabolism by targeting the miR144-3p-PDK4 axis. Cancer Biomark 2021. [Epub ahead of print].

9. Graziano F, Ruzzo A, Giacomini E, et al. Glycolysis gene expression analysis and selective metabolic advantage in the clinical progression of colorectal cancer. Pharmacogenomics J 2017;17:258-64.

10. Blouin JM, Penot G, Collinet M, et al. Butyrate elicits a metabolic switch in human colon cancer cells by targeting the pyruvate dehydrogenase complex. Int J Cancer 2011;128:2591-601.

11. Rajendran VM, Sandle GI. Colonic Potassium Absorption and Secretion in Health and Disease. Compr Physiol 2018;8:1513-36.

12. Liu W, Pan HF, Yang LJ, et al. Panax ginseng C.A. Meyer (Rg3) Ameliorates Gastric Precancerous Lesions in Atp4a-/- Mice via Inhibition of Glycolysis through PI3K/ AKT/miRNA-21 Pathway. Evid Based Complement Alternat Med 2020;2020:2672648.

13. Salami AT, Odukanmi OA, Oshode OO, et al. Modulatory activities of Chrysophyllum albidum and its fractions on microflora and colonic pump activities during inflammatory phase of colitis healing in experimental mice. Food Bioscience 2018;22:38-49.

14. Sachan N, Chandra P, Pal D. Effect of Delonix regia (Boj. Ex Hook.) Raf. stem bark extract against experimentally induced ulcers in rats. Indian J Exp Biol 2017;55:49-54.

15. Sun B, Yuan J, Wang S, et al. Qingchang Suppository Ameliorates Colonic Vascular Permeability in DextranSulfate-Sodium-Induced Colitis. Front Pharmacol 2018;9:1235

16. Tao P, Lin J, Zhang B, et al. Notoginsenoside R2 induces colonic microvascular injuries via regulating the Rap1GAP/PI3K/Akt signaling pathway. Ann Transl Med 2021;9:1743.

17. Schwarz P, Kübler JA, Strnad P, et al. Hepcidin is localised in gastric parietal cells, regulates acid secretion and is induced by Helicobacter pylori infection. Gut 2012;61:193-201.

18. Tao P, Zhang B, Lin J, et al. Thrombospondin-1 aggravates colonic mucosal inflammatory injuries via promoting the differentiation of CD11c+ macrophages with lysosomal activity limited in colitis. Ann Transl Med 2021;9:1738.

19. Zhang X, Zhou C, Miao L, et al. Panax Notoginseng
Protects against Diabetes-Associated Endothelial Dysfunction: Comparison between Ethanolic Extract and Total Saponin. Oxid Med Cell Longev 2021;2021:4722797.

20. Luo H, Vong CT, Tan D, et al. Panax notoginseng Saponins Modulate the Inflammatory Response and Improve IBD-Like Symptoms via TLR/NF-[Formula: see text]B and MAPK Signaling Pathways. Am J Chin Med 2021;49:925-39.

21. Fang C, Guo JW, Wang YJ, et al. Diterbutyl phthalate attenuates osteoarthritis in ACLT mice via suppressing ERK/c-fos/NFATc1 pathway, and subsequently inhibiting subchondral osteoclast fusion. Acta Pharmacol Sin 2021. [Epub ahead of print].

22. Wang S, Tao P, Zhao L, et al. Panax notoginseng Promotes Repair of Colonic Microvascular Injury in Sprague-Dawley Rats with Experimental Colitis. Evid Based Complement Alternat Med 2018;2018:4386571.

23. Chang CS, Liao YC, Huang CT, et al. Identification of a gut microbiota member that ameliorates DSS-induced colitis in intestinal barrier enhanced Dusp6-deficient mice. Cell Rep 2021;37:110016.

24. Yin $\mathrm{Y}, \mathrm{Wu} \mathrm{X}$, Peng B, et al. Curcumin improves necrotising microscopic colitis and cell pyroptosis by activating SIRT1/NRF2 and inhibiting the TLR4 signalling pathway in newborn rats. Innate Immun 2020;26:609-17.

25. Hindryckx P, Devisscher L, Laukens D, et al. Intrarectal administration of oxygenated perfluorodecalin promotes healing of murine colitis by targeting inflammatory hypoxia. Lab Invest 2011;91:1266-76.

26. Falhammar H, Frisén L, Hirschberg AL, et al. Increased Risk of Autoimmune Disorders in 21-Hydroxylase Deficiency: A Swedish Population-Based National Cohort Study. J Endocr Soc 2019;3:1039-52.

27. Hong MY, Henning S, Moro A, et al. Chinese red yeast rice inhibition of prostate tumor growth in SCID mice. Cancer Prev Res (Phila) 2011;4:608-15.

28. Thomas JL, Bose HS. Regulation of human 3-betahydroxysteroid dehydrogenase type-2 (3ßHSD2) by molecular chaperones and the mitochondrial environment affects steroidogenesis. J Steroid Biochem Mol Biol 2015;151:74-84.

Cite this article as: $\mathrm{He} W$, Pan $\mathrm{H}$, Tao P, Lin J, Zhang B, Wang S. Panax notoginseng attenuates hypoxia-induced glycolysis in colonic mucosal epithelial cells in DSS-induced colitis. Ann Transl Med 2022;10(4):218. doi: 10.21037/atm-22566 